\title{
A Machine Learning Access for Selectionof Influential Variables of Several ITK Inhibitors using Regression Research
}

\author{
Rama Devi Chalasani, Radhika Y
}

\begin{abstract}
Introduction: Interleukin-2 inducible T-cell kinase (ITK) is a tyrosine kinase expressed in T-cells, NK cells and mast cells. Selective ITK inhibitors act as an immunosuppressive and anti-inflammatory agent reduces lung inflammation, eosinophil infiltration, and mucous production in response to induced allergic asthma. Methodology: A dataset of 142 ITK inhibitors as dependent variables with 32 properties of compounds as explanatory variables were studied for their multicollinearity prior multivariate regression analysis. After data normalization, an inter-correlation cutoff value of 0.75 resulted in 15 variables and regression analysis resulted in 0.641 $r 2$ and 0.598 adjusted $r 2$ with RMSE 0.634 respectively. As the statistical parameters are within the limits, outlying data was investigated. Results: The standardized residual analysis resulted in nine data points and a new regression model is attempted with $n=133$ and $p=15$ reported improves statistics. Further, stepwise and stepwise AIC regression followed by variance inflation factor analyzed on the dataset revealed only 7 variables as important in defining inhibitory activity of ITK. Permutation and combinations of 7 variables resulted in $r 2$ value $>0.6$ for 5,6 and 7 variables. Hence, to select the best model, FIT criterion was employed where a 5-variable model was judged as best model. Conclusion: Finally, it has been emphasized that increase in HOMO, H-Bond Donors and shape index with a concomitant decrease in number of phenyl groups and LUMO parameter favors ITK inhibition.
\end{abstract}

Keywords -regression, multicollinearity, FIT Kubinyi function, outliers, ITK

\section{INTRODUCTION}

Linear regression is the well-known widely used algorithm in statistics and machine learning. This machine learning regression algorithm can be trained to predict real numbered outputs. Regression analysis on a dataset can be either linear, quadratic, polynomial, non-linear, etc. The regression hypothesis is a function based on relationship between response and explanatory variables to attain statistically significant parameters. Further, selection of appropriate variables in a regression paradigm is an important part of machine learning which refers to the process of reducing the inputs for processing to find the most meaningful, promising variables [1]. The selection reduces number of explanatory variables to describe a response variable [2]. The supervised machine learning processreducesoverfitting because a model with all variables is difficult to interpret, especially when the dimensions of a dataset are large [3].

Revised Manuscript Received on August 19, 2019.

Rama Devi Chalasani, Reaseach Scholar, Department of CSE, GIT, Gitam Deemed to be University. Visakhapatnam.A.P, India

DrRadhika Y, Professor, Department of CSE, GIT, Gitam Deemed to be University. Visakhapatnam.A.P, India.
Based on the dataset, machine learning algorithms might be supervised [4], unsupervised [5] and semi-supervised [6].

In this paper, we provide analytical method to choose influential variables from a dataset of 160 ITK inhibitors with 32 variables that describe the associated properties of these inhibitors in binding to the ITK protein target. Interleukin-2 inducible T-cell kinase (ITK) is a tyrosine kinase that is expressed in T-cells, NK cells and mast cells. Studies suggest that selective ITK inhibitors should be useful as an immunosuppressiveand/or anti-inflammatory agent and reduced lung inflammation, eosinophil infiltration, and mucous production in response to induced allergic asthma [7]. The Tec (tyrosine kinase expressed in hepatocellular carcinoma) family tyrosine kinases play significant roles in mediation of signaling intracellular regions of hematopoietic cells [8]. Due to the critical role of ITK in $\mathrm{T}$ cell development and differentiation, disregulatedITK causes $\mathrm{T}$ cell related disorders.ITKknockout mice displayedcondensed Th2 cells and Th2-type cytokines. These are thought to be important in the inflammatory pathogenic diseases such as allergic asthma and atopic dermatitis [9]. Patients with allergic asthma have increased Th2 cells and Th2 cytokines which lead to lung inflammation [10]. Human immunodeficiency virus (HIV) is a retrovirus causing acquired immunodeficiency syndrome (AIDS). ITK is an important factor in regulation, infection and replication of HIV [11].In vivo experiments with ITK knockout mice suggest a role for ITK inhibitors in the treatment of asthma [12]. Several data from literature reported ITK inhibitors with a focus on achieving broad kinase selectivity as well as good levels of cellular activity [13].

\section{MATERIALS AND METHODS}

\section{a. DATASET}

A dataset of ITK inhibitors that are intended to interact and bind with specific protein target for asthma disease were extracted from literature [14, 15, 16, 17, 18 and 19]. Further, the bio activity data of 142 inhibitory compounds are treated as response (dependent) variable and nearly 32 properties of compounds comprising 2-dimensional and/or 3-dimensional features are considered as explanatory (independent) variables. These variables explain how the response variable is influenced by the change in property values. The 
influence of explanatory variables on response variable can be studied via linear regression accounting that the data is linear. Biological data are usually expressed on a logarithmic scale because of the linear relationship between response and log dose in the mid-region of the log doseresponse curve. Inverse logarithms for activity $(\log 1 / \mathrm{C})$ are used so that higher values are obtained for more effective analogs.

\section{b. EXPLANATORY VARIABLES}

Considering learning problems in data with flexible dimensions, it was reported that not all variables are applicable for predicting the outcome of interest. Some variables might represent negative effect on the accuracy of the model. Nearly 32 variables otherwise referred as independent variables were calculated for each dependent variable. They include: Molecular surface area (MSA), Molecular volume (MV), Molar Refractivity (MR), Kier indices such as Kier Chi 0 (KC0),Kier Chi 1 (KC1), Kier Chi 1 (KC2), Kier Chi 1 (KC3), Kier Chi V0 (KCV0), Kier Chi V1 (KCV1), Kappa 1 index, Kappa 2 index, Kappa 3 index, KAlpha1, KAlpha2, KAlpha3 index, Randic, Balaban, Weiner index, Shape index, Lipinski properties [20] such as Molecular Weight (MW), Hydrogen Bond Acceptors (HBA), Hydrogen Bond Donors (HBD), logP, Number of freely rotatable bonds (RB), Electrostatic properties such as Highest Occupied Molecular Orbital (HOMO), Lowest Unoccupied Molecular Orbital (LUMO), Number of 5-membered rings, 6-membered rings, methyl groups, amino groups, hydroxyl groups and phenyl moieties attached to the main molecule.

\section{c.MULTICOLLINEARITY}

It should be noted that all these physico-chemical properties of ITK inhibitors or few variables should have influential result on the biological activity. Therefore, few variable selection methods have gained prominence. However, it should be noted that few variables display collinearity with other variables. This might result to a situation where a number of independent variables in a multiple regression model are closely correlated to one another. Such mutlicollinearity leads to unreliable and unstable estimates of regression coefficients which results in unstable parameter estimates and makes it very difficult to assess the effect of independent variables on dependent variables.

\section{d. DATA NORMALIZATION}

Prior analysis, data was normalized. Because, there can be instances found in data frame where values for one feature could range between 1-100 and values for other feature might range more than 10000 or so. Such variations in values have greater impact on response variables by that particular feature which would otherwise impact prediction accuracy.

\section{e. REGRESSION ANALYSIS}

The relationship between dependent variable $\left(\log 1 / \mathrm{IC}_{50}\right)$ and independent variables was established by linear regression analysis. Significant variables were chosen based on the statistical data of analysis. Statistical quality of the generated regression equation was judged based on the parameters like correlation coefficient (r), F-value, adjusted r2 etc.

\section{f. OUTLIER ANALYSIS - STANDARDIZED RESIDUALS}

Variance inflation factor (VIF):

Variance inflation factors (VIF) measure how much the variance of the estimated regression coefficients are inflated as compared to when the predictor variables are not linearly related. It is used to explain how much amount multicollinearity (correlation between predictors) exists in a regression analysis. Multicollinearity increases the variance of the regression coefficients. A VIF of four means that the variance (a measure of imprecision) of the estimated coefficients is four times higher because of correlation between the two independent variables. The general rule of thumb is that VIFs exceeding 4 warrant further investigation, while VIFs exceeding 10 are signs of serious multicollinearity requiring correction.

\section{g. FIT KUBINYI FUNCTION}

To define the statistical quality of activity prediction, the number of variables that enter in a regression model are compared by using FIT Kubinyi function [21], which is closely related to $\mathrm{F}$ value.

$$
\mathrm{FIT}=R^{2}(n-k-1) /\left(n+k^{2}\right)\left(1-R^{2}\right)
$$

Wheren is the number of compounds in data set and $k$ is the number of variables in the regression equation.

$\mathrm{F}$ value is sensitive to variations in $\mathrm{k}$, it has low sensitivity when $\mathrm{k}$ is large. Hence, the FIT function is also sensitive towards changes in $\mathrm{k}$, a significantly increase in sensitivity was possible for large $\mathrm{k}$ values [22].

\section{RESULTS AND DISCUSSION}

The ITK molecules extracted from literature are drawn as 2-dimensional structures and selected to calculate physicochemical properties associated with them. These properties referred as independent variables explain the variance in data whereas the dependent variable is the biological activity of chemical compounds that exhibit inhibitory activity against ITK. This activity data, reported as $\mathrm{IC}_{50}$ micro Molar $(\mu \mathrm{M})$ inhibition was the result of bio-analytical outcome. It was observed that to obtain a reliable and robust regression model, it is necessary to contemplate a large data set that covers reasonable chemical diversity and biological activity. Hence, a dataset of 142 ITK inhibitors and their inhibitory activities reported in terms of $\mathrm{IC}_{50}$ in $\mu \mathrm{M}$ were transformed into their corresponding concentration values in order to overcome overlapping data. Data given in Appendix-1.Therefore, to guarantee linear distribution of data, the ITK inhibition values were converted to negative logarithmic values and then used for subsequent analysis Biological data are usually expressed on a logarithmic scale because of the linear relationship between response and log dose in the mid-region of the log dose-response curve. Inverse $\operatorname{logarithms}$ for activity $(\log 1 / \mathrm{C})$ are used so that higher values are obtained for more effective analogs. 
After data normalization, an inter-correlation cutoff value of 0.75 was induced to filter independent variables that are strongly correlated and only variables less than cut off value are selected to perform multicollinear graph. With this cross-limit cut off, out of 32 variables, only 15 variables appeared such as BALABAN ， HBA, HBD, LOGP, RB, LUMO, HOMO, METHYL, AMINO, HYDROXYL, PHENYL, X5.MEM, X6.MEM, KC3 and SHAPE index. The correlation plot given in Fig. 1 suggest that the maximum correlation between variables was around 0.64 between Hydroxyl variable and $\mathrm{KC} 3$ variable only.

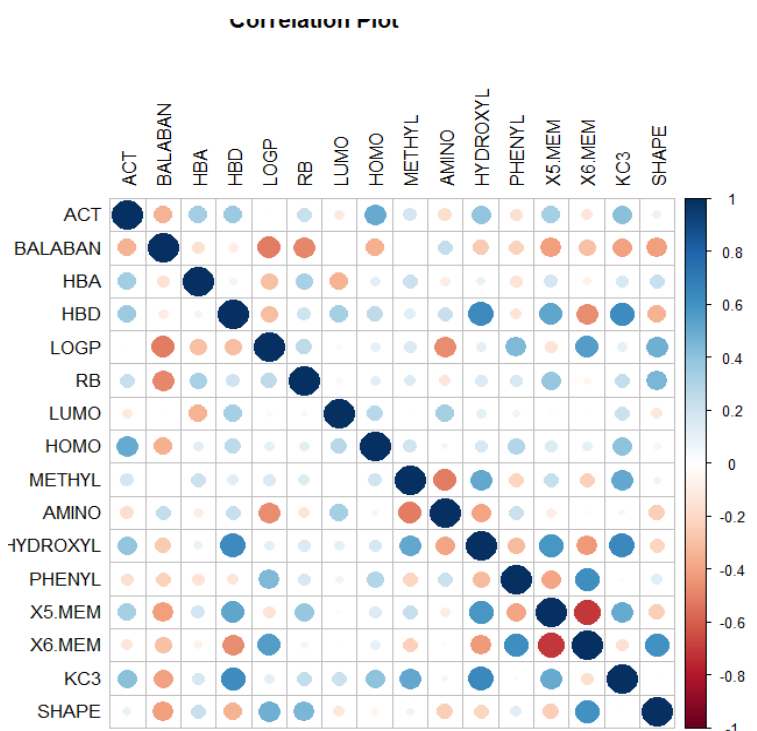

Fig. 1.Correlation plot showing 15 variables under 0.75 inter-correlation.

\section{a. REGRESSION ANALYSIS}

After rejecting few variables that are linearly correlated among one another, a multivariate linear regression analysis was calculated using 142 dependent variables with 15 independent variables. Analysis resulted in $0.641 \mathrm{r} 2$ and 0.598 adjusted $\mathrm{r} 2$ withRMSE 0.634 respectively.

\section{b. MODEL-1:}

$\log (1 / \mathrm{C})=$

-0.352 X BALABAN
+0.154 X HBA
+0.274 X HBD
+0.195 X LOGP
-0.031 X RB
-0.231 X LUMO
+0.600 X HOMO
-0.439 X METHYL
-0.262 X AMINO
-0.090 X HYDROXYL
-0.331 X PHENYL
-0.800 X X5.MEM
-0.863 X X6.MEM
+0.416 X KC3
+0.239 X SHAPE
+0.00
dj $2=0.598 ; F=15.007 ;$

$\mathrm{r}=0.801 ; \mathrm{r} 2=0.641 ;$
RMSE: $0.634 ; \mathrm{n}=142$

As the statistical parameters are within the limits, outlying data was investigated by calculating standardized residuals. Outlying data mask the influential observations and might result in low r2 values. Hence, they should be removed before finalizing the model for analytical prediction.

\section{c. OUTLIER ANALYSIS - STANDARDIZED RESIDUALS}

The standardized residual is a measure of the strength of the difference between observed and expected values. A standardized residual less than -2 and greater than +2 is considered as outlying data point. The standardized residual analysis resulted in nine data points $(44,53,60,66,91,95$, 120,134 and 135) as outlying observations (Table-I).

Table-I: Standardized residuals of dataset.

\begin{tabular}{|c|c|c|c|c|c|}
\hline S. No. & Molecule & Activity & Predicted Activity & Residuals & Standardized Residuals \\
\hline 1 & $2 \_1 . \mathrm{mol}$ & 1.455998 & 0.924238 & 0.53176 & 0.887671 \\
\hline 2 & 2_10a.mol & 0.865309 & 0.528101 & 0.337208 & 0.562904 \\
\hline 3 & 2_10b.mol & 1.596167 & 1.28176 & 0.314407 & 0.524842 \\
\hline 4 & 2_10c.mol & 1.575268 & 1.717554 & -0.14229 & -0.23752 \\
\hline 5 & 2_10d.mol & 1.94786 & 2.352376 & -0.40452 & -0.67526 \\
\hline 6 & 2_10e.mol & 1.32593 & 1.254861 & 0.071069 & 0.118637 \\
\hline 7 & 2_10f.mol & 1.666657 & 1.287395 & 0.379262 & 0.633105 \\
\hline 8 & 2_10g.mol & 1.440394 & 1.039435 & 0.400959 & 0.669324 \\
\hline 9 & 2_10h.mol & 1.93957 & 1.679835 & 0.259735 & 0.433578 \\
\hline 10 & 2_10i.mol & 1.9243 & 1.862195 & 0.062104 & 0.103671 \\
\hline 11 & 2_10j.mol & 1.617825 & 1.666916 & -0.04909 & -0.08195 \\
\hline 12 & 2_9a.mol & -0.45477 & 0.340897 & -0.79567 & -1.32821 \\
\hline 13 & 2_9b.mol & 0.699782 & 1.093644 & -0.39386 & -0.65748 \\
\hline 14 & 2_9c.mol & 0.844013 & 1.36435 & -0.52034 & -0.8686 \\
\hline 15 & 5_1.mol & -0.43776 & -0.68382 & 0.24606 & 0.410749 \\
\hline 16 & 5_17.mol & -1.05751 & -1.58886 & 0.531353 & 0.886992 \\
\hline 17 & 5_18.mol & -1.65394 & -1.50639 & -0.14755 & -0.24631 \\
\hline
\end{tabular}


A Machine Learning Access for Selectionof Influential Variables of Several ITK Inhibitors using Regression Research

\begin{tabular}{|c|c|c|c|c|c|}
\hline 18 & 5_19.mol & -0.95911 & -0.94845 & -0.01066 & -0.0178 \\
\hline 19 & 5_20.mol & -0.87533 & -1.19931 & 0.323976 & 0.540816 \\
\hline 20 & 5_21.mol & -0.28933 & 0.50621 & -0.79554 & -1.328 \\
\hline 21 & 5_22.mol & -0.45121 & -0.74662 & 0.295411 & 0.493131 \\
\hline 22 & 5_23.mol & -0.76778 & -1.02663 & 0.258847 & 0.432095 \\
\hline 23 & 5_24.mol & -2.10022 & -2.02904 & -0.07119 & -0.11883 \\
\hline 24 & 5_25.mol & -0.1241 & -0.90584 & 0.781741 & 1.304966 \\
\hline 25 & 5_26.mol & -0.19106 & -0.25132 & 0.060258 & 0.100589 \\
\hline 26 & 5_30.mol & -2.76148 & -2.51981 & -0.24167 & -0.40342 \\
\hline 27 & 5_31.mol & -1.10065 & -1.45719 & 0.356536 & 0.595168 \\
\hline 28 & 5_32.mol & -1.43778 & -0.72426 & -0.71352 & -1.19108 \\
\hline 29 & 5_33.mol & -0.3702 & -0.74553 & 0.375324 & 0.626532 \\
\hline 30 & 5_34.mol & -0.92071 & -0.02231 & -0.8984 & -1.4997 \\
\hline 31 & 5_35.mol & -0.99136 & -1.56055 & 0.569193 & 0.950158 \\
\hline 32 & 5_36.mol & -0.9337 & -0.61895 & -0.31475 & -0.52542 \\
\hline 33 & 5_37.mol & -0.83454 & -0.59207 & -0.24247 & -0.40475 \\
\hline 34 & 5_38.mol & -0.66233 & -1.2522 & 0.589873 & 0.984679 \\
\hline 35 & 5_39.mol & -0.57808 & -0.67513 & 0.097052 & 0.16201 \\
\hline 36 & 5_40.mol & -0.15562 & -0.31904 & 0.163416 & 0.272791 \\
\hline 37 & 5_42.mol & -1.40214 & -1.18419 & -0.21795 & -0.36382 \\
\hline 38 & 5_43.mol & -1.74995 & -1.3746 & -0.37536 & -0.62658 \\
\hline 39 & 5_44.mol & -0.68836 & -0.47958 & -0.20877 & -0.34851 \\
\hline 40 & 5_45.mol & -0.33041 & -0.48964 & 0.159224 & 0.265794 \\
\hline 41 & 5_46.mol & -1.53538 & -0.82806 & -0.70732 & -1.18073 \\
\hline 42 & 5_47.mol & -1.40112 & -0.74776 & -0.65336 & -1.09066 \\
\hline 43 & 5_48.mol & -1.59813 & -1.04433 & -0.5538 & -0.92447 \\
\hline 44 & 5_49.mol & -2.71496 & -1.34343 & -1.37153 & -2.28951 \\
\hline 45 & 7_10.mol & 0.819647 & 0.51534 & 0.304307 & 0.507982 \\
\hline 46 & 7_11.mol & 0.33342 & 0.46586 & -0.13244 & -0.22108 \\
\hline 47 & 7_12.mol & 0.565119 & -0.05097 & 0.616089 & 1.028442 \\
\hline 48 & 7_13.mol & 0.778494 & 0.568191 & 0.210303 & 0.351061 \\
\hline 49 & 7_14.mol & 1.346048 & 0.618007 & 0.728041 & 1.215325 \\
\hline 50 & 7_15.mol & 1.242997 & 0.580755 & 0.662242 & 1.105486 \\
\hline 51 & 7_16.mol & 0.774379 & 0.07883 & 0.695549 & 1.161085 \\
\hline 52 & 7_17.mol & 0.68019 & -0.0756 & 0.755791 & 1.261648 \\
\hline 53 & 7_18.mol & -2.20389 & -0.5813 & -1.62259 & -2.7086 \\
\hline 54 & 7_19.mol & -0.09801 & -0.20899 & 0.110973 & 0.185249 \\
\hline 55 & 7_2.mol & 0.084854 & 0.738427 & -0.65357 & -1.09101 \\
\hline 56 & 7_20.mol & 0.144795 & -0.18517 & 0.329968 & 0.550819 \\
\hline 57 & 7_21.mol & 1.253203 & 0.44961 & 0.803593 & 1.341444 \\
\hline 58 & 7_22.mol & 1.253203 & 0.618526 & 0.634677 & 1.059471 \\
\hline 59 & 7_23.mol & -0.22876 & 0.354739 & -0.5835 & -0.97403 \\
\hline 60 & 7_24.mol & -0.91796 & 0.524736 & -1.4427 & -2.40831 \\
\hline 61 & 7_28.mol & -0.85636 & 0.055968 & -0.91232 & -1.52295 \\
\hline 62 & 7_29.mol & 0.553659 & 0.477098 & 0.076561 & 0.127804 \\
\hline 63 & 7_31.mol & 0.165362 & -0.05747 & 0.222832 & 0.371975 \\
\hline 64 & 7_32.mol & 1.008796 & 0.044239 & 0.964556 & 1.610141 \\
\hline 65 & 7_33.mol & -0.41424 & 0.078623 & -0.49286 & -0.82274 \\
\hline 66 & 7_34.mol & -1.47229 & 0.014329 & -1.48662 & -2.48162 \\
\hline 67 & 7_36.mol & 0.535905 & 0.282073 & 0.253832 & 0.423724 \\
\hline 68 & 7_37.mol & -0.23155 & 0.063739 & -0.29529 & -0.49293 \\
\hline 69 & 7_38.mol & 0.819156 & 0.774194 & 0.044962 & 0.075056 \\
\hline 70 & 7_39.mol & 0.576216 & 0.079262 & 0.496954 & 0.829569 \\
\hline 71 & 7_41.mol & -0.21135 & 0.397473 & -0.60883 & -1.01632 \\
\hline 72 & 7_42.mol & 1.067359 & 0.687754 & 0.379605 & 0.633677 \\
\hline
\end{tabular}


International Journal of Recent Technology and Engineering (IJRTE) ISSN: 2277-3878, Volume-8 Issue-2S8, August 2019

\begin{tabular}{|c|c|c|c|c|c|}
\hline 73 & 7_43.mol & 1.067408 & 1.012418 & 0.05499 & 0.091795 \\
\hline 74 & 7_44.mol & 0.392457 & 0.442659 & -0.0502 & -0.0838 \\
\hline 75 & 7_47.mol & 0.948987 & 0.474873 & 0.474114 & 0.791442 \\
\hline 76 & 7_48.mol & -0.64896 & -0.54862 & -0.10034 & -0.1675 \\
\hline 77 & 7_49.mol & -0.0567 & -0.27141 & 0.214707 & 0.358412 \\
\hline 78 & 7_50.mol & -0.9478 & -0.09476 & -0.85304 & -1.42399 \\
\hline 79 & 7_51.mol & -0.77126 & -0.23855 & -0.53271 & -0.88926 \\
\hline 80 & 7_52.mol & 0.039652 & 1.148741 & -1.10909 & -1.85141 \\
\hline 81 & 7_53.mol & 0.926738 & 1.108006 & -0.18127 & -0.30259 \\
\hline 82 & 7_54.mol & 0.428418 & -0.05608 & 0.4845 & 0.808779 \\
\hline 83 & 7_56.mol & 1.401823 & 0.661563 & 0.74026 & 1.235722 \\
\hline 84 & 7_8.mol & 1.164873 & 0.713536 & 0.451336 & 0.753419 \\
\hline 85 & 7_9.mol & 0.81848 & 0.715032 & 0.103448 & 0.172686 \\
\hline 86 & 9_10.mol & 0.161972 & -0.06232 & 0.224295 & 0.374417 \\
\hline 87 & 9_11.mol & -0.24178 & -0.45079 & 0.209008 & 0.348899 \\
\hline 88 & 9_12.mol & -0.46119 & 0.289279 & -0.75047 & -1.25277 \\
\hline 89 & 9_15.mol & -0.00467 & -0.39178 & 0.387106 & 0.646199 \\
\hline 90 & 9_16.mol & 0.436105 & -0.33236 & 0.768467 & 1.282808 \\
\hline 91 & 9_17.mol & -1.73634 & -0.34392 & -1.39243 & -2.32439 \\
\hline 92 & 9_18.mol & 0.034477 & -0.25329 & 0.287763 & 0.480364 \\
\hline 93 & 9_19.mol & 0.160176 & -0.42944 & 0.589611 & 0.984243 \\
\hline 94 & 9_2.mol & 0.005809 & -0.19907 & 0.20488 & 0.342008 \\
\hline 95 & 9_20.mol & 1.085817 & -0.16952 & 1.255334 & 2.095538 \\
\hline 96 & 9_21.mol & 0.066416 & 0.3734 & -0.30698 & -0.51245 \\
\hline 97 & 9_22.mol & 0.187642 & -0.34064 & 0.52828 & 0.881861 \\
\hline 98 & 9_23.mol & 0.29708 & 0.298276 & -0.0012 & -0.002 \\
\hline 99 & 9_24.mol & -0.00751 & -0.31953 & 0.312018 & 0.520855 \\
\hline 100 & 9_25.mol & 0.062907 & 0.355823 & -0.29292 & -0.48897 \\
\hline 101 & 9_26.mol & 1.480931 & 0.741714 & 0.739217 & 1.233981 \\
\hline 102 & 9_7.mol & -0.56774 & -0.75082 & 0.183083 & 0.305621 \\
\hline 103 & 9_8.mol & 0.033181 & 0.309048 & -0.27587 & -0.46051 \\
\hline 104 & 9_9.mol & 0.006916 & 0.724425 & -0.71751 & -1.19774 \\
\hline 105 & 11_1.mol & -0.67822 & -0.83585 & 0.157622 & 0.26312 \\
\hline 106 & 11_11.mol & -1.64626 & -0.96827 & -0.67799 & -1.13177 \\
\hline 107 & 11_12.mol & -2.09295 & -1.56173 & -0.53122 & -0.88677 \\
\hline 108 & 11_13.mol & -0.40356 & -1.30667 & 0.903116 & 1.507578 \\
\hline 109 & 11_14.mol & -0.10015 & -0.25564 & 0.155488 & 0.259558 \\
\hline 110 & 11_15.mol & -0.09117 & -0.18115 & 0.089975 & 0.150196 \\
\hline 111 & 11_16.mol & -1.01492 & -0.12399 & -0.89093 & -1.48724 \\
\hline 112 & 11_17.mol & -0.51824 & -0.17582 & -0.34242 & -0.5716 \\
\hline 113 & 11_18.mol & -1.14089 & -0.35141 & -0.78948 & -1.31788 \\
\hline 114 & 11_19.mol & -0.63799 & -0.26466 & -0.37333 & -0.6232 \\
\hline 115 & 11_20.mol & -0.66753 & -0.2078 & -0.45973 & -0.76743 \\
\hline 116 & 11_21.mol & -0.36388 & -0.42232 & 0.058445 & 0.097563 \\
\hline 117 & 11_22.mol & 0.595849 & 0.156118 & 0.439732 & 0.734047 \\
\hline 118 & 11_23.mol & 0.514635 & 0.261046 & 0.253588 & 0.423317 \\
\hline 119 & 11_24.mol & 0.570415 & 0.13848 & 0.431934 & 0.721031 \\
\hline 120 & 11_25.mol & 1.608065 & 0.348714 & 1.25935 & 2.102244 \\
\hline 121 & 11_26.mol & 0.587767 & -0.05956 & 0.647324 & 1.080583 \\
\hline 122 & 11_27.mol & 0.374709 & 0.062217 & 0.312493 & 0.521646 \\
\hline 123 & 11_28.mol & -0.18076 & -0.00097 & -0.17979 & -0.30013 \\
\hline 124 & 11_29.mol & -0.65207 & 0.048318 & -0.70038 & -1.16916 \\
\hline 125 & 11_30.mol & 0.050845 & 0.176954 & -0.12611 & -0.21051 \\
\hline 126 & 11_31.mol & 0.023748 & -0.31239 & 0.336141 & 0.561124 \\
\hline 127 & 11_32.mol & 0.394283 & 0.425002 & -0.03072 & -0.05128 \\
\hline
\end{tabular}


A Machine Learning Access for Selectionof Influential Variables of Several ITK Inhibitors using Regression Research

\begin{tabular}{|l|l|c|c|c|c|}
\hline 128 & $11 \_35 . m o l$ & 0.851132 & 0.409948 & 0.441184 & 0.736472 \\
\hline 129 & $11 \_38 . m o l$ & 0.985855 & 0.305881 & 0.679975 & 1.135087 \\
\hline 130 & $11 \_39 . m o l$ & 0.670739 & 0.477098 & 0.193641 & 0.323246 \\
\hline 131 & $11 \_40 . m o l$ & 0.705558 & 0.665709 & 0.039849 & 0.06652 \\
\hline 132 & $11 \_41 . m o l$ & 1.568291 & 0.502729 & 1.065561 & 1.77875 \\
\hline 133 & $11 \_43 . m o l$ & -0.3469 & 0.35156 & -0.69846 & -1.16595 \\
\hline 134 & $11 \_44 . m o l$ & -1.15927 & 0.37295 & -1.53222 & -2.55775 \\
\hline 135 & $11 \_45 . m o l$ & -0.68003 & 0.672343 & -1.35237 & -2.25753 \\
\hline 136 & $11 \_46 . m o l$ & -0.31596 & 0.172223 & -0.48818 & -0.81493 \\
\hline 137 & $11 \_47 . m o l$ & 0.817092 & 1.076509 & -0.25942 & -0.43305 \\
\hline 138 & $11 \_48 . m o l$ & 1.330439 & 0.591719 & 0.738719 & 1.23315 \\
\hline 139 & $11 \_6 . m o l$ & 0.472511 & -0.3201 & 0.792613 & 1.323115 \\
\hline 140 & $11 \_7 . m o l$ & 0.512377 & -0.31287 & 0.825243 & 1.377585 \\
\hline 141 & $11 \_8 . m o l$ & -0.6827 & -0.70677 & 0.024074 & 0.040187 \\
\hline 142 & $11 \_9 . m o l$ & 0.236413 & -0.30659 & 0.543005 & 0.906442 \\
\hline & & & Standard Deviation & 0.599051 & \\
\hline
\end{tabular}

From the outlier study, nine outliers should be removed from analysis in order to guarantee linear distribution of correlations among parameters. By excluding these data points, regression analysis should be carried out to assess statistical significance. Therefore, a new regression model is attempted by excluding nine data points $(44,53,60,66$, $91,95,120,134$ and 135) being detected as outliers with data being $n=133$ and $\mathrm{p}=15$; and from the result given in model-2, it was evidenced that an improvement was observed in $\mathrm{r} 2$ value from 0.641 to 0.739 and adjusted $\mathrm{r} 2$ reported improved value from 0.598 to 0.706 , respectively.

d. MODEL-2:

\begin{tabular}{|c|c|}
\hline $\log (1 / C)=$ & $\begin{array}{l}-0.257 \text { X BALABAN } \\
+0.210 \text { X HBA } \\
+0.360 \text { X HBD } \\
+0.268 \text { X LOGP } \\
-0.131 \text { X RB } \\
-0.148 \text { X LUMO } \\
+0.674 \text { X HOMO } \\
-0.432 \text { X METHYL } \\
-0.259 \text { X AMINO } \\
-0.129 \text { X HYDROXYL } \\
-0.306 \text { X PHENYL } \\
-0.678 \text { X X5.MEM } \\
-0.832 \text { X X6.MEM } \\
+0.342 \text { X KC3 } \\
+0.370 \text { X SHAPE } \\
+0.00\end{array}$ \\
\hline
\end{tabular}

$\mathrm{r}=0.860 ; \mathrm{r} 2=0.739 ;$ adj $\mathrm{r} 2=0.706 ; \mathrm{F}=22.095$;

RMSE: $0.543 ; n=133$

e. STEPWISE REGRESSION
Further, in order to delineate most important variables that would efficiently estimate coefficients of data are identified using step-wise regression method [23] by entering and removing variables based on $\mathrm{p}$ values, in a stepwise manner until there is no variable left to enter or remove any more. A variable will enter the model if $\mathrm{p}$ value less than 0.1 and any variable will be removed if $p$ is $>0.3$ by default. Therefore, from 15 variables, only the following five variables were found to be important in defining the model with accuracy.

f. MODEL-3:

$\log (1 / \mathrm{C})=$

$$
\begin{aligned}
& +0.390 \text { X HBD } \\
& -0.296 \text { X LUMO } \\
& +0.683 \text { X HOMO } \\
& -0.327 \text { X PHENYL } \\
& +0.321 \text { X SHAPE } \\
& +0.00
\end{aligned}
$$$$
\mathrm{r}=0.818 ; \mathrm{r} 2=0.669 ; \operatorname{adj} \mathrm{r} 2=0.656 ; \mathrm{F}=51.24 ;
$$$$
\text { RMSE: } 0.587 ; n=133
$$

Based on F-value, it can be stated that model-3 reported better statistic variables and to confirm stepwise selection method, step-wise AIC regression was performed.

\section{g. STEPWISE AIC REGRESSION}

Stepwise AIC regression builds regression model from a set of 15 candidate independent variables by entering and removing predictors based on akaike information criteria [24], in a stepwise manner until there is no variable left to enter or remove any more. Analysis resulted in eleven variable model and the statistics are tabulated in Table-II. 
Table-II: Stepwise Summary

\begin{tabular}{|c|c|c|c|c|c|c|}
\hline Variable & Method & AIC & $R S S$ & SUM SQ & $R-S Q$ & $A D J . R-S Q$ \\
\hline HOMO & Addition & 327.779 & 87.520 & 44.480 & 0.33697 & 0.33191 \\
\hline PHENYL & Addition & 302.140 & 71.098 & 6.9 .2 & 0.46138 & 0.45310 \\
\hline SHAPE & Addition & 288.230 & 63.082 & 68.918 & 0.52211 & 0.51099 \\
\hline HDB & Addition & 268.420 & 53.541 & 78.459 & 0.59439 & 0.58171 \\
\hline LUMO & Addition & 243.553 & 43.747 & 88.253 & 0.66858 & 0.65553 \\
\hline METHYL & Addition & 242.702 & 42.820 & 89.180 & 0.67561 & 0.66016 \\
\hline AMINO & Addition & 240.300 & 41.426 & 90.574 & 0.68617 & 0.66859 \\
\hline KC3 & Addition & 239.515 & 40.567 & 91.433 & 0.69267 & 0.67284 \\
\hline X5.MEM & Addition & 238.965 & 39.797 & 92.203 & 0.69851 & 0.67645 \\
\hline X6.MEM & Addition & 233.695 & 37.680 & 94.320 & 0.71455 & 0.69115 \\
\hline BALABAN & Addition & 231.656 & 36.553 & 95.447 & 0.72308 & 0.69791 \\
\hline
\end{tabular}

From the step-wise summary, it can be observed that few regression models can be obtained with combination of variables such as for a 5-variable model, the $\mathrm{r} 2$ value is 0.669 which is similar to the result obtained from stepwise regression analysis based on $\mathrm{p}$ value. Further, a 6-variable model showed $0.675 \mathrm{r} 2$, a 7 -variable model has 0.686 with an increasing $\mathrm{r} 2$ observed for 8 -variable model (0.692), 9-variable model resulted in 0.698 and 10, 11variable models showed 0.714 and 0.723 r2 values, respectively.

Variance inflation factors are evaluated for all 15 variables of model2. From Table-III, it is evidenced that the variables BALABAN, X5.MEM and X6.MEM have high VIF values and hence are excluded from regression analysis.

Table-III: Variance inflation factors of all 15 variables in the study

\begin{tabular}{|c|l|c|c|}
\hline SNO & Variables & Tolerance & VIF \\
\hline 1 & BALABAN & $0.079 \underline{4}$ & 12.6 \\
\hline 2 & HBA & 0.318 & 3.14 \\
\hline 3 & HBD & 0.213 & 4.71 \\
\hline 4 & LOGP & 0.159 & 6.27 \\
\hline 5 & RB & 0.162 & 6.18 \\
\hline 6 & LUMO & 0.470 & 2.13 \\
\hline 7 & HOMO & 0.590 & 1.69 \\
\hline 8 & METHYL & 0.189 & 5.30 \\
\hline 9 & AMINO & 0.169 & 5.90 \\
\hline 10 & HYDROXYL & 0.154 & 6.48 \\
\hline 11 & PHENYL & 0.170 & 5.87 \\
\hline 12 & X5.MEM & $0.049 \underline{3}$ & 20.3 \\
\hline 13 & X6.MEM & $0.034 \underline{2}$ & 29.2 \\
\hline 14 & KC3 & 0.118 & 8.45 \\
\hline 15 & SHAPE & 0.135 & 7.43 \\
\hline
\end{tabular}

A regression model with the remaining 12 variables resulted in better predicted statistics, however, few variables are found to be not significant statistically, where $\mathrm{p}$ value is greater than 0.1. Removing variables such as LOGP, RB, AMINO, HYDROXYL and KC3 and a new regression model attempted with 7 variableshas improved F-statistic parameter from 23.54 to 37.33 .

h. MODEL-4: $\log (1 / \mathrm{C})=$

+0.034 X HBA

$+0.394 \times$ X HBD

-0.289 X LUMO

$+0.700 \times$ X HOMO

-0.093 X METHYL

-0.348 X PHENYL

+0.324 X SHAPE

$+0.00$

$\mathrm{r}=0.822 ; \mathrm{r} 2=0.676 ;$ adj $\mathrm{r} 2=0.658 ; \mathrm{F}=37.33$;

RMSE: $0.584 ; \mathrm{n}=133$

Moreover, several permutation and combination of 7 variables from model4 were carried out using olsrr package which resulted in several combinations with $\mathrm{r} 2$ value $>0.6$ for 5, 6 and 7 variables (Fig. 2).Upon careful observation of the above graph, it is evident that combination of variables for a:5-variable model numbered 99 resulted in $\mathrm{r} 2: 0.669$ and adjusted $\mathrm{r} 20.656$, 6-variable model 120 displayed r2: 0.676 and adjusted r2 0.660 whereas a 7 -variable model numbered 127 has $\mathrm{r} 2$ : 0.676 and adjusted r2 0.658 , respectively. Regression data and statistics given below.

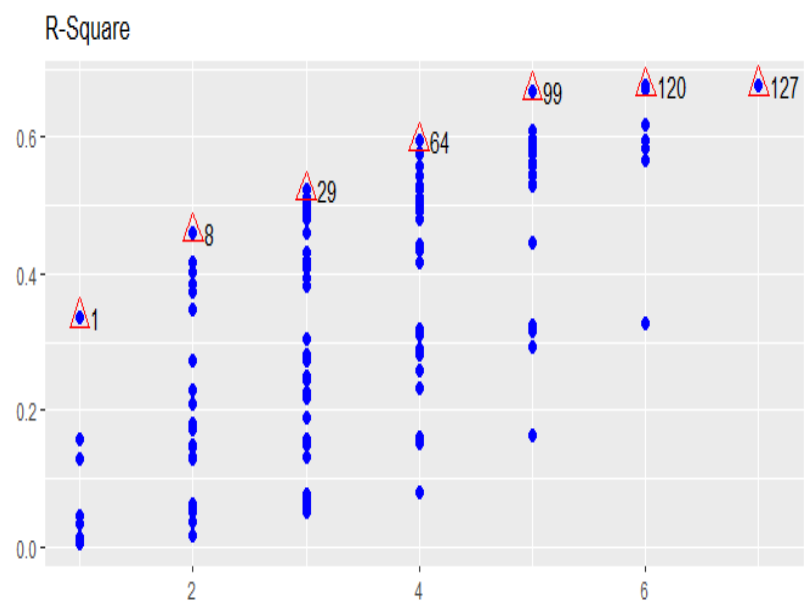

Fig. 2. Panel of fit criteria for all possible regression models 
A Machine Learning Access for Selectionof Influential Variables of Several ITK Inhibitors using Regression Research

i. MODEL-5: 5-VARIABLE MODEL

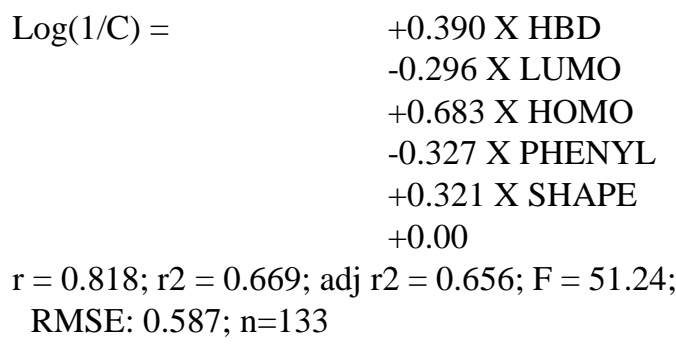

j. MODEL-6: 6-VARIABLE MODEL

$$
\begin{array}{cl}
\log (1 / \mathrm{C})= & +0.401 \mathrm{X} \text { HBD } \\
& -0.303 \text { X LUMO } \\
& +0.709 \text { X HOMO } \\
& -0.091 \text { X METHYL } \\
& -0.354 \text { X PHENYL } \\
& +0.334 \text { X SHAPE } \\
& +0.00 \\
\text { r }=0.822 ; \mathrm{r} 2=0.676 ; \text { adj } \mathrm{r} 2=0.660 ; \mathrm{F}=43.73 ; \\
\text { RMSE: } 0.583 ; \mathrm{n}=133
\end{array}
$$

\section{k. MODEL-7: 7-VARIABLE MODEL}

$$
\begin{array}{cl}
\log (1 / \mathrm{C})= & +0.034 \mathrm{X} \mathrm{HBA} \\
& +0.394 \text { X HBD } \\
& -0.290 \text { X LUMO } \\
& +0.700 \text { X HOMO } \\
& -0.094 \text { X METHYL } \\
& -0.348 \text { X PHENYL } \\
& +0.325 \times \text { SHAPE } \\
& +0.00 \\
\text { r }=0.822 ; \mathrm{r} 2=0.676 ; \text { adj } \mathrm{r} 2 & =0.658 ; \mathrm{F}=37.33 ; \\
\text { RMSE: } 0.585 ; \mathrm{n}=133 &
\end{array}
$$

The Table-IV given below presents the best possible models generated from several combinations of variables and the various statistical parameters obtained from analysis suggest that all models are within the limits of validation parameters of regression. It was observed that a low RMSE value was obtained for a 6-variable model whereas significant F-value reported by a 5-variable model. Hence, to select the best model, FIT criterion was employed.

Table-IV: Statistical data and validation parameter values of 5, 6 and 7 variable model equations.

\begin{tabular}{|l|l|l|l|}
\hline \multirow{2}{*}{ Variable } & \multicolumn{3}{|l|}{ Coefficient } \\
\cline { 2 - 4 } & $\begin{array}{l}\text { 5- } \\
\text { variable } \\
\text { model }\end{array}$ & $\begin{array}{l}\text { 6- } \\
\text { variable } \\
\text { model }\end{array}$ & $\begin{array}{l}\text { 7- } \\
\text { variable } \\
\text { model }\end{array}$ \\
\hline HBA & - & - & +0.034 \\
\hline HBD & +0.390 & +0.401 & +0.394 \\
\hline LUMO & -0.296 & -0.303 & -0.290 \\
\hline HOMO & +0.683 & +0.709 & +0.700 \\
\hline METHYL & - & -0.091 & -0.094 \\
\hline PHENYL & -0.327 & -0.354 & -0.348 \\
\hline SHAPE & +0.321 & +0.334 & +0.325 \\
\hline Intercept & -0.00 & -0.00 & -0.00 \\
\hline Statistics & \multicolumn{5}{|l}{} \\
\hline$R$ & 0.818 & 0.822 & 0.822 \\
\hline$r^{2}$ & 0.669 & 0.676 & 0.676 \\
\hline Adj $r^{2}$ & 0.656 & 0.660 & 0.658 \\
\hline \multicolumn{4}{|l}{} \\
\hline
\end{tabular}

RMSE: Root Mean Square Error

MSE: Mean Square Error

MAE: Mean Absolute Error

\section{l. FIT KUBINYI FUNCTION}

The best model was judged based on the high value obtained from FIT function and hence regression models with five, six and seven variables are applied to select best possible model (Table-V).

Table-V: FIT function of three regression models obtained for five, six and seven variables.

\begin{tabular}{|c|l|l|l|l|}
\hline $\begin{array}{c}\text { Variables } \\
(\mathbf{k})\end{array}$ & $\boldsymbol{r}^{2}$ & $\boldsymbol{s}$ & $\boldsymbol{F}$ & FIT \\
\hline 5 & 0.669 & 0.254 & 51.24 & 1.624 \\
\hline 6 & 0.676 & 0.255 & 43.73 & 1.555 \\
\hline 7 & 0.676 & 0.256 & 37.33 & 1.432 \\
\hline
\end{tabular}

The statistical FIT values of the models reported inTable-5 suggest that the model with five variables represents best model since this model has high FIT than others. Therefore, further analysis was carried out with five-variable model. The actual and predicted value of this model is given in Figure. 3.

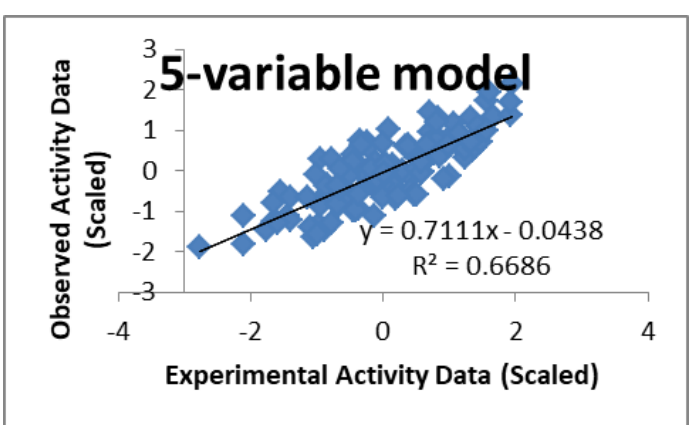

Fig. 3. Regression plot of 5-variable model data with $\mathrm{n}=133$ showing $\mathrm{r} \mathbf{2} \mathbf{0 . 6 6 9}$

After rejecting nine outliers from the data set, a prominent increase in R-squared and adjusted R-squared values along with F-statistic were observed which suggests the fact that an improved regression diagnostics was achieved by five variables viz., Hydrogen Bond Donors (HBD), HOMO (Highest Occupied Molecular Orbital), LUMO (Lowest Unoccupied Molecular Orbital), No. of Phenyl groups and Shape index features in defining ITK inhibitor activity.

The highest occupied and lowest unoccupied molecular orbitals (HOMO and LUMO) are vital in envisaging the molecule reactivity. HOMO is the outmost orbital comprising the electron and LUMO is the first orbital

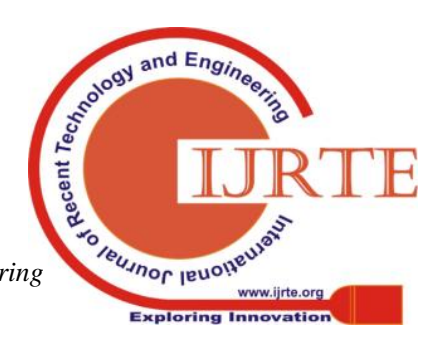


which is devoid of any electron. The HOMO energy measures the electron donating character and LUMO measures its electron accepting character [25]. Based on the coefficients of 5-variable model, a high value of LUMO energy contributes negatively to the activity. Electron-withdrawing substituents, such as halogens, lower the energy of LUMO. Hence, ITK inhibitors with electron-withdrawing substituents improve activity. From the coefficient values, it can be emphasized that an increase in HOMO, H-Bond Donors and shape index is required to inhibit ITK by these set of compounds whereas on the other hand, reduced number of phenyl groups and LUMO parameter favors ITK inhibition.

\section{CONCLUSION}

Variable selection as a method of choice to choose most important variables which could describe the inhibitory properties of nearly 142 ligands tested against ITK protein target against 32 independent variables was studied using multivariate regression technique. With inter correlation cut off value of 0.75 resulted in 15 variables and regression resulted in $0.641 \mathrm{r} 2$ and 0.598 adjusted r2 with RMSE 0.634 respectively. Eliminating nine outlying data evidenced an improvement in $\mathrm{r} 2$ value to 0.739 and adjusted $\mathrm{r} 2$ reported 0.706 , whereas stepwise regression suggested only five variables as important in defining the model based on $\mathrm{p}$ values. Finally based on stepwise AIC regression and variance inflation factor analytics resulted in 7 variables. Several permutation and combination of these 7 variables reported $\mathrm{r} 2$ value $>0.6$ for 5,6 and 7 variables and the statistical FIT values of all the three models suggested 5variable model as best model since this model has high FIT than others. From the coefficient data, increase in HOMO, H-Bond Donors and shape index inhibits ITK whereas decrease in the number of phenyl groups and LUMO parameter favors ITK inhibition.

\section{REFERENCES}

1. Forman G. An extensive empirical study of feature selection metrics for text classification, J. Mach. Learn. Res., 2003, vol. 3 (pg. 1289-1305.

2. Guyon I, Elisseeff A. An introduction to variable and feature selection, J. Mach Learn Res., 2003, vol. 3 (pg. 1157-1182).

3. E. Candes and T. Tao. The dantzig selector: statistical estimation when $\mathrm{p}$ is much larger than $\mathrm{n}$. The Annals of Statistics, pages 2313-2351, 2007.

4. J. Weston, A. Elisseff, B. Schoelkopf, and M. Tipping. Use of the zero norm with linear models and kernel methods. Journal of Machine Learning Research, 3:1439-1461, 2003.

5. J.G. Dy and C.E. Brodley. Feature selection for unsupervised learning. The Journal of Machine Learning Research, 5:845-889, 2004.

6. Z. Zhao and H. Liu. Semi-supervised feature selection via spectral analysis. In Proceedings of SIAM International Conference on Data Mining, 2007.

7. Mueller, C.; August, A. J. Immunol. 2003, 170, 5056] [Kanner, S. B.; Perez-Villar, J. J. Trends Immunol. 2003, 24, 249.

8. Tec family kinases in $\mathrm{T}$ lymphocyte development and function. Berg LJ, Finkelstein LD, Lucas JA, Schwartzberg PL. Annu Rev Immunol. 2005; 23():549-600.
9. Attenuation of immunological symptoms of allergic asthma in mice lacking the tyrosine kinase ITK. Mueller C, August A. J Immunol. 2003 May 15; 170(10):5056-63.

10. Reduced airway hyperresponsiveness and tracheal responses during allergic asthma in mice lacking tyrosine kinase inducible T-cell kinase. Ferrara TJ, Mueller C, Sahu N, Ben-Jebria A, August A. J Allergy ClinImmunol. 2006 Apr; 117(4):780-6.

11. Selective targeting of ITK blocks multiple steps of HIV replication. Readinger JA, Schiralli GM, Jiang JK, Thomas CJ, August A, Henderson AJ, Schwartzberg PL. ProcNatlAcadSci U S A. 2008 May 6; 105(18):6684-9.

12. August A.; Ragin M. J. Regulation of T-cell responses and diseases by Tec kinase ITK. Int. Rev. Immumol. 2012, 31, $155-165$.

13. Das J.; Furch J. A.; Liu C.; Moquin R. V.; Lin J.; Spergel S. H.; McIntyre K. W.; Shuster D. J.; O’Day K. D.; Penhallow B.; Hung C. Y.; Doweyko A. M.; Kamath A.; Zhang H.; Marathe P.; Kanner S. B.; Lin T. A.; Dodd J. H.; Barrish J. C.; Wityak J. Discovery and SAR of 2-amino-5(thioaryl)thiazoles as potent and selective ITK inhibitors. Bioorg. Med. Chem. Lett. 2006, 16, 3706-3712.

14. Doris Riether et al. 5-Aminomethylbenzimdazoles as potent ITK antagonists. Bioorganic \& Medicinal Chemistry Letters 19 (2009) 1588-1591.

15. Ho Yin Lo et al. 2-Aminobenzimidazoles as potent ITK antagonists: trans-stilbene-like moieties targeting the kinase specificity pocket. Bioorganic \& Medicinal Chemistry Letters 18 (2008) 6218-6221.

16. Roger J. Snow et al. Hit-to-lead studies on benzimidazole inhibitors of ITK: Discovery of a novel class of kinase inhibitors. Bioorganic \& Medicinal Chemistry Letters 17 (2007) 3660-3665

17. Kevin J. Moriarty et al. Discovery, SAR and X-ray structure of 1H-benzimidazole-5-carboxylic acid cyclohexyl-methyl-amides as inhibitors of inducible T-cell kinase (ITK). Bioorganic \& Medicinal Chemistry Letters 18 (2008) 5545-5549.

18. Michael P. Winters et al. 5-Aminomethyl-1Hbenzimidazoles as orally active inhibitors of inducible Tcell kinase (ITK).Bioorganic \& Medicinal Chemistry Letters 18 (2008) 5541-5544.

19. Brian N. Cook et al. Discovery of potent inhibitors of interleukin-2 inducible T-cell kinase (ITK) through structure-based drug design. Bioorganic \& Medicinal Chemistry Letters 19 (2009) 773-777.

20. CA Lipinski, F Lombardo, BW Dominy and P J Feeney, Experimental and computational approaches to estimate solubility and permeability in drug discovery and development settings, Adv Drug Del Rev 46 3-26 (2001).

21. Kubinyi H. Variable selection in QSAR studies. II. A Highly Efficient Combination of Systematic Search and Evolution. Quant. Struct. Act. Relat.13, 1994, 393-401.

22. Kubinyi H. Variable selection in QSAR studies. I. An evolutionary algorithm. Quant. Struct. Act. Relat. 13, 1994, 285-294.

23. Chatterjee, Samprit and Hadi, Ali. Regression Analysis by Example. 5th ed. N.p.: John Wiley \& Sons, 2012. Print.

24. Venables, W. N. and Ripley, B. D. (2002) Modern Applied Statistics with S. Fourth edition. Springer.

25. Hall LH, Mohney B, Kier LB. The Electrotopological State: Structure Information at the Atomic Level for Molecular Graphs. J. Chem. Inf. Comput. Sci. 31, 1991, 76-82. 